\title{
Novel gene discovery for hearing loss and other routes to increased diagnostic rates
}

\author{
Hannie Kremer ${ }^{1,2,3}$
}

Received: 20 June 2021 / Accepted: 15 September 2021 / Published online: 1 October 2021

(c) The Author(s) 2021

\begin{abstract}
Despite decades of research, there is much to be learned about the genetic landscape of sensorineural hearing loss. Novel genes for hearing loss remain to be identified while 'secrets' of the known genes need to be uncovered. These 'secrets' include regulatory mechanisms of gene activity and novel aspects of gene structure. To obtain a more complete picture of the genetics of hearing loss, the available experimental and bioinformatic tools need to be fully exploited. This is also true for data resources such as ENCODE. For the inner ear, however, such data resources and analytical tools need to be developed or extended. Collaborative studies provide opportunities to achieve this and to optimally use those tools and resources that are already available. This will accelerate the discoveries that are necessary for improving molecular genetic diagnostics and genetic counselling and for the development of therapeutic strategies.
\end{abstract}

\section{Introduction}

It is clear from the missing diagnoses in molecular genetic testing as well as from deafness loci for which the defective genes are still elusive that our knowledge of the genetic landscape of (nonsyndromic) sensorineural hearing loss is far from complete (Hereditary Hearing Loss Homepage (HHL Homepage, https://hereditaryhearingloss.org/); Vona $\mathrm{B}$ et al. 2019). The main reasons for missing defects underlying hearing loss with a presumed genetic cause include limitations of the employed technologies and of tools for variant interpretation, as well as our incomplete knowledge of the structure and regulatory mechanisms of genes already known to be associated with hearing loss. A further important reason for missing diagnoses in routine molecular genetic testing is that the catalogue of genes known to be

Hannie Kremer

Hannie.Kremer@radboudumc.nl

1 Hearing and Genes, Department of Otorhinolaryngology, Radboud University Medical Center, Nijmegen, The Netherlands

2 Department of Human Genetics, Radboud University Medical Center, Internal Postal Code 855, P.O. Box 9101, 6500 HB Nijmegen, The Netherlands

3 Donders Institute for Brain, Cognition and Behaviour, Radboud University Medical Center, Nijmegen, The Netherlands associated with hearing loss is incomplete. This is confirmed by a steady pace of gene discoveries for hearing loss (HHL Homepage; Vona et al. 2019). However, besides that deafness loci can harbour elusive genetic defects, it should also be considered that a locus resulted from innocent methodological mapping errors, genetic heterogeneity in families, or phenocopies among affected family members.

\section{Identification of novel genes for hearing loss}

It is an open question how many genes for hearing loss await to be identified. For mouse, it is estimated that about 1000 genes are critical for hearing (Ingham et al. 2019) which suggests a comparable exceptional genetic heterogeneity for hearing loss in humans, as well. How should we effectively complete the gene catalogue for hearing loss? The predominant strategy for the genes that have been identified since early 2019, was whole exome or genome sequencing (WES, WGS) in multiple members of families with hearing loss segregating in an autosomal dominant or recessive pattern. This was preceded or complemented by linkage analysis or homozygosity mapping in several of the studies and functional effects of variants were demonstrated in cell models. To confirm that the genes were indeed associated with hearing loss, either animal models were studied (mouse, zebrafish, fruitfly) or cohorts of index cases were screened to identify additional 
pathogenic variants. A second important approach was screening of index cases for variants in candidate genes that were found to be critical for hearing in knock out mice from the International Mouse Phenotyping Consortium (IMPC; Dickinson et al. 2016). Such candidate genes have also been employed to prioritise variants identified in family studies. Ideally, both animal models and cohort studies are combined to confirm the association of a gene with a hearing loss phenotype. In addition, in vitro or ex vivo experiments, biochemical assays, and/or cell biological assays can provide evidence for pathogenicity of variants in novel genes for hearing loss.

Multiplex families and candidate genes are indeed excellent sources for the identification of novel genes for hearing loss (see article by Acharya, this issue). As there is a positive correlation between the number of investigated genes and the diagnostic yield (Vona et al. 2019), it is important to accelerate gene identification and to confirm associations of those genes for which the evidence is insufficient for reporting their variants in molecular genetic testing. Decreasing costs of genome analyses allow to increase the numbers of index cases and families that can be studied. Importantly, this creates excellent opportunities to make major steps forward in large collaborative studies as such studies reveal study cohort(s) and datasets with sufficient power for novel analysis strategies. Innovative meta-analysis tools can be applied that need to be either specifically designed or adapted for hearing loss. Meta-analyses have been successfully employed to identify genes for intellectual disability although the predominance of de novo mutations in this condition reduces the complexity of such an analysis (Kaplanis et al. 2020; Lelieveld et al. 2016). Large cohorts will also allow their stratification for specific analyses e.g. based on the presumed inheritance pattern of the condition and functional effect of causative variants (e.g. loss of function effect in recessively inherited hearing loss), or onset age and characteristics of the hearing loss to finetune metaanalysis strategies or enrich for specific genetic defects. A further benefit of joining forces is that this provides opportunities to implement cost-effective candidate gene analysis and pre-screening strategies of the known genes for hearing loss. This is relevant for those cases that were tested previously only for a subset of genes and for cases for which the possibilities for genetic testing are limited. Unsolved cases can subsequently enlarge patient cohorts for further studies. In a recently established consortium, called Solve-RD, both data and expertise are shared to diagnose patients with rare diseases (Zurek et al. 2021). Solve-RD could serve as a blueprint for a consortium on hearing loss. Funding to establish a consortium for hearing loss would be an excellent way to provide more research groups and/or diagnostic laboratories access to WES and WGS. The importance to collaborate and to apply WES or WGS also provides opportunities to urge significantly increased resources for research in countries and communities in which these are too limited.

\section{Scrutinising (known) genes for hearing loss}

An important open question is whether identification of novel genes for hearing loss or scrutinising the known genes will have the largest impact on the diagnostic rates. Several types of genetic variation of these known genes are currently not (effectively) addressed and/or difficult to interpret. This is specifically the case for variants in the noncoding regions of genes and flanking regulatory regions including sequences that determine (tissue-specific) chromatin organisation. Tools to predict aberrant transcript splicing caused by (deep-)intronic variants are being used and predicted effects are often experimentally tested in mini-gene splice assays. Evaluation of variants in noncoding DNA for a potential deleterious effect on gene regulation is more challenging. Modelling of variants in conserved sequences in mouse can provide insights into a (potentially) pathogenic effect and mechanism (see article by Bowl, this issue). This is also illustrated for a small deletion in $H G F$ intron 5 that was predicted to occur in the 3'UTR of an alternatively spliced transcript (Morell et al. 2020). Such extensive studies are only possible for a limited number of variants, and likely to be initiated if there are strong indications for deleteriousness. Our insight into the regulatory landscape of gene expression is limited, especially for the inner ear, which hampers variant interpretation. Emerging resources and tools such as the ENCODE (the Encyclopedia of DNA Elements) dataset (ENCODE; Project Consortium et al. 2020), and DESCARTES (the Developmental Single Cell Atlas of Gene Regulation; Cao et al. 2020; Domcke et al. 2020) will increasingly facilitate to address variants in regulatory regions, although the inner ear was not analysed yet in these projects. In other projects, studies of the inner ear are being performed and data on gene expression and chromatin accessibility are becoming available (Muthu et al. 2019; Yizhar-Barnea et al. 2018; gEAR portal [https://umgear. org/]). An experimental strategy to obtain clues to potentially pathogenic effects of noncoding variation on either transcript splicing or gene regulation is to combine WGS and RNA sequencing data of affected subjects. However, this is only meaningful for those genes that are expressed at a sufficient level in accessibly cell types such as fibroblasts and peripheral blood cells. In the latter cell source, this is true for about $30 \%$ of the known genes for hearing loss (the Genotype-Tissue Expression (GTEx) project and K. Neveling, personal communication). Analysis pipelines for this strategy have been developed and successfully applied e.g. for mitochondriopathy patients (Kremer et al. 2017). This approach can also shed light on potential effects of (missense 
or synonymous) variants of unknown significance (VUS) on transcript splicing or of structural variants such as inversions and translocations that are often missed in short read sequencing. Such structural variants can, however, be effectively identified by long read genome sequencing and Bionano optical mapping (Chan et al. 2018). Obviously, inner ear-specific effects on transcript splicing and gene expression will not be detected with the indicated approaches. Options to elucidate such effects are iPSC-derived inner ear cells that are being more broadly implemented (see article by Romano, this issue). The relevance of technologies in molecular genetic testing for hearing loss is reviewed in de Bruijn et al. (2021).

In the Deafness Variation Database, more than $79 \%$ of variants in genes implicated in hearing loss are classified as VUS, most of which are missense variants (Azaiez et al. 2018), despite the availability of a plethora of tools to predict potential deleterious effects. Obviously, developing strategies to evaluate the pathogenicity of these VUS may reveal a major increase of the diagnostic yield. Addressing enrichment of such variants in large (population-specific) cohorts of index cases as compared to controls can provide valuable insights but many variants are ultra-rare. Analysing family members for segregation patterns of such variants remains important but is often difficult. Studying potential enrichment of variants in specific gene regions might enhance variant interpretation. Also, bioinformatic tools such as MetaDome (Wiel et al. 2019) can be explored for their effectivity in variant interpretation in genes for hearing loss or for selection of missense variants to ultimately perform functional evaluation at the protein level. Such functional assays still need to be developed for most of the hearing loss associated genes which is not a trivial task (see article by Miyoshi, this issue). The same is true for the implementation of such assays in clinical practice.

Last but not least, variants in known genes for hearing loss with an allele frequency higher than expected based on disease incidence or prevalence need to be considered for a pathogenic effect with incomplete penetrance. This might be specifically relevant for genetic types of hearing loss with a variable, mainly adult onset and/or variable severity. The occurrence of reduced penetrance of variants as well as the potential genetic complexity of a Mendelian disorder is exemplified in clinically and allelically highly heterogeneous $A B C A 4$-associated retinal degeneration (Cremers et al. 2020). The genetic complexity is illustrated by an extremely hypomorphic missense variant with an allele frequency of $\sim 7 \%$ in Europe which is completely penetrant when present in cis with an additional variant. Also, the existence of (non-)genetic modifiers is indicated part of which might have a gender-specific effect on disease penetrance.

All discussed aspects for the evaluation of variants in genes already known to be associated with hearing loss are also relevant for the identification of novel genes for hearing loss. Therefore, the knowledge and expertise gained by analysing the full range of genetic variation of these known genes will facilitate the identification of novel genes that are critical for hearing in humans. The analysis of known genes as well as the identification of novel genes for hearing loss can be importantly accelerated and supported by initiatives such as building population-specific databases for hearing loss and the development of hearing loss-specific multi-omics baseline data.

\section{Non-Mendelian inheritance patterns in hearing loss}

Large WES and/or WGS datasets of genetically unexplained index cases as well as families will enable us to address non-Mendelian inheritance patterns of hearing loss, more specifically digenic or oligogenic inheritance. So far, there is no strong evidence of digenic inheritance of hearing loss. However, non-Mendelian inheritance might well significantly contribute to hearing loss in isolated cases for which the diagnostic yield is significantly lower than for familial cases. This is also true for adult-onset hearing loss even if this is familial. The genes involved might already be associated with hearing loss but could also be novel. The recently developed platform ORVAL (the Oligogenic Resource for Variant AnaLysis) aims to identify candidate pathogenic variant combinations in gene pairs (Renaux et al. 2019) and it would be interesting to explore this for hearing loss.

In conclusion, identification of novel genes for hearing loss is an ongoing process that is importantly facilitated by the developments in DNA and RNA sequencing technologies and in tools for bioinformatic and experimental analysis of genetic variation, as well as by the emerging resources of $e$.g. regulatory elements. The same technologies and tools will also facilitate the identification and interpretation of variation in the known genes for hearing loss and non-Mendelian inheritance patterns. Important steps forward can be made in global collaborative frameworks designed to exchange expertise, enlarge patient cohorts and combine datasets to increase the power of analysis tools that are already available or to be developed. The ongoing efforts to characterise the genetic landscape of hearing loss in many different populations, as presented in several articles in this issue (e.g. Naz, this issue) are already paving the way for global collaborative efforts.

Funding The author did not receive support from any organisation for the submitted work. 


\section{Declarations}

Conflict of interest The author has no conflicts of interest or competing interests to declare.

Data availability Not applicable.

Code availability Not applicable.

Open Access This article is licensed under a Creative Commons Attribution 4.0 International License, which permits use, sharing, adaptation, distribution and reproduction in any medium or format, as long as you give appropriate credit to the original author(s) and the source, provide a link to the Creative Commons licence, and indicate if changes were made. The images or other third party material in this article are included in the article's Creative Commons licence, unless indicated otherwise in a credit line to the material. If material is not included in the article's Creative Commons licence and your intended use is not permitted by statutory regulation or exceeds the permitted use, you will need to obtain permission directly from the copyright holder. To view a copy of this licence, visit http://creativecommons.org/licenses/by/4.0/.

\section{References}

Azaiez H, Booth KT, Ephraim SS, Crone B, Black-Ziegelbein EA, Marini RJ, Shearer AE, Sloan-Heggen CM, Kolbe D, Casavant T, Schnieders MJ, Nishimura C, Braun T, Smith RJH (2018) Genomic landscape and mutational signatures of deafness-associated genes. Am J Hum Genet 103:484-497. https://doi.org/10. 1016/j.ajhg.2018.08.006

Cao J, O’Day DR, Pliner HA, Kingsley PD, Deng M, Daza RM, Zager MA, Aldinger KA, Blecher-Gonen R, Zhang F, Spielmann M, Palis J, Doherty D, Steemers FJ, Glass IA, Trapnell C, Shendure J (2020) A human cell atlas of fetal gene expression. Science 370:eaba7721. https://doi.org/10.1126/science.aba7721

Chan S, Lam E, Saghbini M, Bocklandt S, Hastie A, Cao H, Holmlin E, Borodkin M (2018) Structural variation detection and analysis using bionano optical mapping. Methods Mol Biol 1833:193-203. https://doi.org/10.1007/978-1-4939-8666-8_16

Cremers FPM, Lee W, Collin RWJ, Allikmets R (2020) Clinical spectrum, genetic complexity and therapeutic approaches for retinal disease caused by $A B C A 4$ mutations. Prog Retin Eye Res 79:100861. https://doi.org/10.1016/j.preteyeres.2020.100861

de Bruijn SE, Fadaie Z, Cremers FPM, Kremer H, Roosing S (2021) The impact of modern technologies on molecular diagnostic success rates, with a focus on inherited retinal dystrophy and hearing loss. Int J Mol Sci 22:2943. https://doi.org/10.3390/ijms22062943

Dickinson ME, Flenniken AM, Ji X, Teboul L, Wong MD et al (2016) High-throughput discovery of novel developmental phenotypes. Nature 537:508-514. https://doi.org/10.1038/nature19356

Domcke S, Hill AJ, Daza RM, Cao J, O’Day DR, Pliner HA, Aldinger KA, Pokholok D, Zhang F, Milbank JH, Zager MA, Glass IA, Steemers FJ, Doherty D, Trapnell C, Cusanovich DA, Shendure J (2020) A human cell atlas of fetal chromatin accessibility. Science 370:7612. https://doi.org/10.1126/science.aba7612

ENCODE Project Consortium, Moore JE, Purcaro MJ, Pratt HE, Epstein CB et al (2020) Expanded encyclopaedias of DNA elements in the human and mouse genomes. Nature 583:699-710. https://doi.org/10.1038/s41586-020-2493-4
Ingham NJ, Pearson SA, Vancollie VE, Rook V, Lewis MA, Chen J, Buniello A, Martelletti E, Preite L, Lam CC, Weiss FD, Powis Z, Suwannarat P, Lelliott CJ, Dawson SJ, White JK, Steel KP (2019) Mouse screen reveals multiple new genes underlying mouse and human hearing loss. PLoS Biol 17:e3000194. https://doi.org/10. 1371/journal.pbio.3000194

Kaplanis J, Samocha KE, Wiel L, Zhang Z et al (2020) Evidence for 28 genetic disorders discovered by combining healthcare and research data. Nature 586:757-762. https://doi.org/10.1038/ s41586-020-2832-5

Kremer LS, Bader DM, Mertes C, Kopajtich R et al (2017) Genetic diagnosis of Mendelian disorders via RNA sequencing. Nat Commun 8:15824. https://doi.org/10.1038/ncomms15824

Lelieveld SH, Reijnders MR, Pfundt R, Yntema HG, Kamsteeg EJ, de Vries P, de Vries BB, Willemsen MH, Kleefstra T, Löhner K, Vreeburg M, Stevens SJ, van der Burgt I, Bongers EM, Stegmann AP, Rump P, Rinne T, Nelen MR, Veltman JA, Vissers LE, Brunner HG, Gilissen C (2016) Meta-analysis of 2,104 trios provides support for 10 new genes for intellectual disability. Nat Neurosci 19:1194-1196. https://doi.org/10.1038/nn.4352

Morell RJ, Olszewski R, Tona R, Leitess S, Wafa TT, Taukulis I, Schultz JM, Thomason EJ, Richards K, Whitley BN, Hill C, Saunders T, Starost MF, Fitzgerald T, Wilson E, Ohyama T, Friedman TB, Hoa M (2020) Noncoding microdeletion in mouse $h g f$ disrupts neural crest migration into the stria vascularis, reduces the endocochlear potential, and suggests the neuropathology for human nonsyndromic deafness DFNB39. J Neurosci 40:29762992. https://doi.org/10.1523/JNEUROSCI.2278-19.2020

Muthu V, Rohacek AM, Yao Y, Rakowiecki SM, Brown AS, Zhao YT, Meyers J, Won KJ, Ramdas S, Brown CD, Peterson KA, Epstein DJ (2019) Genomic architecture of Shh-dependent cochlear morphogenesis. Development 46:dev181339. https://doi.org/10.1242/ dev.181339

Renaux A, Papadimitriou S, Versbraegen N, Nachtegael C, Boutry S, Nowé A, Smits G, Lenaerts T (2019) ORVAL: a novel platform for the prediction and exploration of disease-causing oligogenic variant combinations. Nucleic Acids Res 47(W1):W93-W98. https://doi.org/10.1093/nar/gkz437

Vona B, Müller M, Dofek S, Holderried M, Löwenheim H, Tropitzsch A (2019) A Big Data perspective on the genomics of hearing loss. Laryngo-Rhino-Otol 98:S58-S81. https://doi.org/10. 1055/a-0803-6149

Wiel L, Baakman C, Gilissen D, Veltman JA, Vriend G, Gilissen C (2019) MetaDome: Pathogenicity analysis of genetic variants through aggregation of homologous human protein domains. Hum Mutat 40:1030-1038. https://doi.org/10.1002/humu.23798

Yizhar-Barnea O, Valensisi C, Jayavelu ND, Kishore K, Andrus C, Koffler-Brill T, Ushakov K, Perl K, Noy Y, Bhonker Y, Pelizzola M, Hawkins RD, Avraham KB (2018) DNA methylation dynamics during embryonic development and postnatal maturation of the mouse auditory sensory epithelium. Sci Rep 8:17348. https:// doi.org/10.1038/s41598-018-35587-x

Zurek B, Ellwanger K, Vissers LELM, Schüle R et al (2021) Solve-RD: systematic pan-European data sharing and collaborative analysis to solve rare diseases. Eur J Hum Genet. https://doi.org/10.1038/ s41431-021-00859-0

Publisher's Note Springer Nature remains neutral with regard to jurisdictional claims in published maps and institutional affiliations. 